\title{
On The Propagation And Modulation Of Electrostatic Solitary Waves Observed Near The Magnetopause On Cluster
}

\author{
J. S. Pickett ${ }^{1}$, I. W. Christopher ${ }^{1}$, B. Grison ${ }^{2}$, S. Grimald ${ }^{3}$, O. Santolík ${ }^{2,4}$, P. M. E. \\ Décréau $^{5}$, B. Lefebvre ${ }^{6}$, M. J. Engebretson ${ }^{7}$, L. M. Kistler ${ }^{6}$, D. Constantinescu ${ }^{8}$, \\ L.-J. Chen ${ }^{6}$, Y. Omura ${ }^{9}$, G. S. Lakhina ${ }^{10}$, D. A. Gurnett ${ }^{1}$, N. Cornilleau-Wehrlin ${ }^{11}$, \\ A. N. Fazakerley ${ }^{12}$, I. Dandouras ${ }^{4}$, and E. Lucek ${ }^{13}$
}

${ }^{1}$ Department of Physics and Astronomy, The University of Iowa, Iowa City, IA, USA

${ }^{2}$ Institute of Atmospheric Physics, Prague, Czech Republic

${ }^{3}$ Université de Toulouse, Toulouse, France

${ }^{4}$ Charles University, Faculty of Mathematics and Physics, Prague, Czech Republic

${ }^{5}$ LPC2E, CNRS et Université d'Orléans, Orléans, France

${ }^{6}$ Space Science Center, University of New Hampshire, Durham, NH, USA

${ }^{7}$ Dept. of Physics, Augsburg College, Minneapolis, MN, USA

${ }^{8}$ TU Braunschweig, Braunschweig, Germany

${ }^{9}$ Research Institute for Sustainable Humanosphere, Kyoto University,UJI, Kyoto, Japan

${ }^{10}$ Upper Atmospheric Studies, Indian Institute of Geomagnetism, Navi Mumbai, India

${ }^{11}$ Station de Radioastronomie de Nançay, Observatoire de Paris, CNRS, Nançay, France

${ }^{12}$ Mullard Space Science Laboratory, Holmbury St. Mary, UK

${ }^{13}$ Imperial College, London, UK

\begin{abstract}
We present the results of a study of Electrostatic Solitary Waves (ESWs) in which propagation of a series of noncyclical ESWs is observed from one Cluster spacecraft to another over distances as great as tens of $\mathrm{km}$ and time lags as great as a few tens of ms. This propagation study was conducted for locations near the magnetopause on the magnetosheath side. Propagation was found primarily toward the earth with speeds on the order of 1500 to $2400 \mathrm{~km} / \mathrm{s}$. The sizes of the ESWs obtained from these velocities were on the order of $1 \mathrm{~km}$ along the magnetic field direction and several tens of $\mathrm{km}$ perpendicular. These results are consistent with measurements on single spacecraft in which the ESW propagation is observed with time lags of only $\sim 0.1 \mathrm{~ms}$. Our results thus show the stability of ESWs over time periods much greater than their own characteristic pulse durations of a few 100s of microseconds. We present also the results of a study of ESW modulation at the magnetopause on the earthward side. We found that ESWs were modulated at $\sim 1.3 \mathrm{~Hz}$, consistent with a Pc1 wave which was observed concurrently. During this time, tens of eV electron beams are present. We propose a Buneman type instability in which the $\mathrm{E}_{/ /}$component of the Pc1 waves provides a mechanism for accelerating electrons, resulting in the generation of the ESWs modulated at the Pc1 frequency.
\end{abstract}

Keywords: Magnetopause, Electrostatic Solitary Waves, Pc1 Waves, Buneman Instability PACS: $94.30 . \mathrm{ch}$, 94.05.Fg, 94.30.Ms, 94.05.Pt, 94.30.cq

\section{INTRODUCTION}

Electrostatic Solitary Waves (ESWs) have been highly studied since their first observations in the data of S3-3 [1] and with the advent in the 1990s of digital high time resolution waveform receivers mounted on spacecraft (c.f., [2]). ESWs are generally observed in these waveform data as isolated pulses and as a series of cyclic or noncyclic pulses or wavepackets, usually with similar amplitudes and pulse time durations. They are usually found in regions of space which are turbulent or in which there are mixing of plasmas, such as in and near boundary layers and along auroral field lines [3]. Much of the past work, both observational and theoretical, on ESWs and their association with electron phase space holes, has been 
discussed by Franz et al. [4] in the introduction to their work on the properties of small-amplitude electron phase-space holes observed on the Polar spacecraft. In addition Ghosh et al. [5] also reviewed many of the past ESW studies in the introduction to their work on electron acoustic solitary waves in a magnetized plasma with application to boundary layers. ESWs are almost always found in and near the magnetopause and its boundary as first reported by Cattell et al. [6] through Polar observations at the subsolar, equatorial magnetopause. The ESWs in this region were reported by them to have amplitudes up to $\sim 25 \mathrm{mV} / \mathrm{m}$ and velocities from $\sim 150 \mathrm{~km} / \mathrm{s}$ to $>2000 \mathrm{~km} / \mathrm{s}$ with scale sizes on the order of a kilometer (comparable to the Debye length). ESWs and amplitude modulated electrostatic waves (AMEWs) were later found by Matsumoto et al. [7] to be associated with 3dimensional reconnection using data from GEOTAIL as the spacecraft skimmed along the dayside magnetopause. They showed that the ESWs and AMEWs were not random noises but rather nonlinear coherent structures, possibly providing dissipation in the electron diffusion region.

In the present study, we use data obtained on the Cluster spacecraft near the magnetopause boundary layer (MPBL) to further our understanding of ESWs in this very dynamic region. We first present data relating to the propagation of ESWs close to the magnetopause on the magnetosheath side. For this study, we use the multi-spacecraft aspect of Cluster to determine ESW propagation speed and size. The results of this study are presented in Section 2. We follow this in Section 3 with a study of ESWs which are observed to occur in bursts modulated at a low frequency of approximately $1.3 \mathrm{~Hz}$. The Cluster observations presented in Sections 2 and 3 were obtained by the Wideband Data (WBD) plasma wave receiver [8], the Spatio-Temporal Analysis of Field Fluctuations Search Coil (STAFF-SC) and Spectrum 2002/02/19

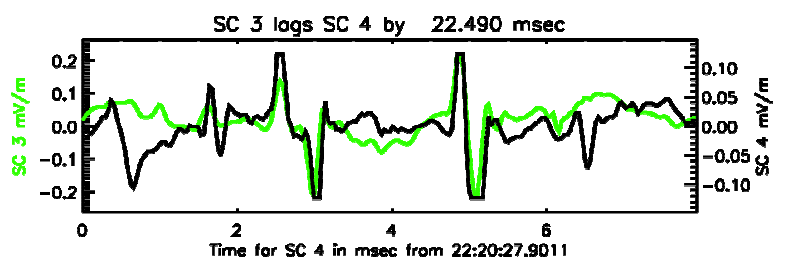

FIGURE 1. Example of ESW propagation from Cluster SC4 to SC3 where the SC3 waveforms have been brought forward in time by the detected lag to SC4 $(22.49 \mathrm{~ms})$ and overlaid on the SC4 waveforms. (Extracted from Figure 5 of Pickett et al., [16])
Analyzer (STAFF-SA) instruments [9], the Fluxgate Magnetometer (FGM) [10], and the WHISPER resonance sounder and wave analyzer [11]. In Section 4 we discuss the significance of the observations contained in Sections 2 and 3 with respect to the physical processes that are likely to be behind the generation of the ESWs and their modulation. In addition for the ESW modulation event we discuss the characteristics of the electrons using the PEACE data [12] and of the ions using the CIS data [13]. Finally, we summarize our results and present some basic conclusions in Section 5.

\section{PROPAGATION OF ESWS}

Prior to the launch of Cluster, spacecraft observations of the propagation of ESWs near the magnetopause were constrained to a single spacecraft. The technique of observing and determining propagation involved the use of interferometry. A dipole antenna, which spans the length from one probe to another through the spacecraft body, is divided into two antennas with the spacecraft body acting as the end probe for each of the two. An ESW is first detected by one of these two antennas. The delay between that measurement and the detection of the ESW at the second antenna is then used, together with the known antenna lengths, to get the propagation speed (c.f., [14]). The measurements are usually oriented with respect to the background magnetic field to determine if the propagation was along the field or at some angle off this direction. In most cases, the ESWs are seen propagating along the magnetic field direction, either parallel or anti-parallel. However, the maximum distance traveled by ESWs with these measurements was limited by the length of the antennas, thus being on the order of $50 \mathrm{~m}$. With the advent of Cluster, it was now possible to consider propagation from one spacecraft to another. There have been two documented cases of ESW propagation on Cluster: 1) along auroral field lines at a distance of 4.8 $\mathrm{R}_{\mathrm{E}}$ on the nightside [15], and 2) in the dayside magnetopause boundary layer at a distance of $11.9 \mathrm{R}_{\mathrm{E}}$ on the magnetosheath side [16]. The method for determination of cross spacecraft propagation is a complex and time consuming process of defining the shape (time duration and amplitude) characteristics of the ESWs, using a computer program to search for these signatures in the data from each spacecraft, and then cross-correlating the ESW detections across the various spacecraft using a series of irregularly spaced ESWs (see $[15,16]$ ) for more details on this method). In Figure 1, which we have extracted from Figure 5 of Pickett et al. [16], we show the $8 \mathrm{~ms}$ waveforms from Cluster spacecraft 4 (SC4) as a black line with the 
waveforms from SC3 overlaid as a green line and brought forward in time by its lag to SC4 by $22.49 \mathrm{~ms}$. The agreement is quite striking and shows that it is possible for ESWs to be stable over a $22 \mathrm{~ms}$ time span, allowing for the calculation of the propagation speed and size of the ESWs similar to the method employed on one spacecraft.

Using this same method, a few other cases of propagation in the MPBL on the magnetosheath side were found in the Cluster data set in the only period of time when the Cluster spacecraft were on the dayside near the MPBL and the spacecraft were close enough to observe the propagation. This was the approximate February through May 2002 period when the interspacecraft separations were on the order of $100 \mathrm{~km}$, with a perfect tetrahedron targeted for the northern cusp.

TABLE 1. Characteristics of ESWs obtained through cross spacecraft waveform correlations

\begin{tabular}{|c|c|c|c|c|c|c|c|c|c|c|}
\hline $\begin{array}{l}\text { Date } \\
\text { (Year/Month/ } \\
\text { Day) }\end{array}$ & Time (UT) & $\begin{array}{l}\text { Cluster } \\
\text { space- } \\
\text { craft }\end{array}$ & $\begin{array}{l}\text { Lag } \\
\text { (ms) }\end{array}$ & $\begin{array}{l}\text { Pulse } \\
\text { Duration } \\
\text { (ms) }\end{array}$ & $\begin{array}{l}\mathrm{D}_{\mathrm{B} / /} \\
(\mathrm{km})\end{array}$ & $\begin{array}{l}D_{B \perp} \\
(\mathbf{k m})\end{array}$ & $\begin{array}{l}\text { Velocity } \\
(\mathrm{km} / \mathrm{s})\end{array}$ & $\begin{array}{l}\text { Direction } \\
\text { (referenced } \\
\text { to Earth) }\end{array}$ & $\begin{array}{l}\mathrm{L}_{\mathrm{B} / /} \\
(\mathrm{km})\end{array}$ & $\begin{array}{l}\mathrm{L}_{\mathrm{B} \perp} \\
(\mathrm{km})\end{array}$ \\
\hline $\begin{array}{c}\text { 2002/02/19 } \\
11.84 \mathrm{R}_{\mathrm{E}} \\
\text { 13.53 MLT } \\
\text {-53.9 MLat }\end{array}$ & $22: 20: 27.90$ & 4,3 & 22.5 & 0.60 & 30 & 40 & 1,334 & Away & 0.8 & $\geq 40$ \\
\hline $\begin{array}{l}2002 / 04 / 03 \\
11.79 \mathrm{R}_{\mathrm{E}} \\
10.85 \mathrm{MLT} \\
-52.5 \mathrm{MLat}\end{array}$ & $18: 00: 54.54$ & 2,1 & 19.5 & 0.30 & 38 & 95 & 1,947 & Toward & 0.6 & $\geq 95$ \\
\hline $\begin{array}{c}2002 / 05 / 04 \\
13.32 R_{\mathrm{E}} \\
08.25 \text { MLT } \\
\text {-42.4 MLat }\end{array}$ & $14: 02: 57.11$ & 4,1 & 37.8 & 0.25 & 71 & 79 & 1,870 & Toward & 0.5 & $\geq 79$ \\
\hline $\begin{array}{c}2002 / 05 / 04 \\
13.26 \mathrm{R}_{\mathrm{F}} \\
\text { 08.27 MLT } \\
\text {-42.6 MLat }\end{array}$ & 14:08:06.19 & 3,1 & 15.6 & 0.30 & 37 & 97 & 2,365 & Toward & 0.7 & $\geq 97$ \\
\hline $\begin{array}{c}2002 / 05 / 04 \\
13.26 \mathrm{R}_{\mathrm{E}} \\
08.27 \text { MLT } \\
-42.6 \text { MLat }\end{array}$ & $14: 08: 06.20$ & 3,1 & 17.0 & 0.45 & 37 & 97 & 2,167 & Toward & 1.0 & $\geq 97$ \\
\hline $\begin{array}{l}2002 / 05 / 04 \\
13.26 R_{\mathrm{E}} \\
08.27 \text { MLT } \\
\text {-42.6 MLat }\end{array}$ & $14: 08: 06.23$ & 3,1 & 21.4 & 0.37 & 37 & 97 & 1,724 & Toward & 0.6 & $\geq 97$ \\
\hline
\end{tabular}

Table 1 shows the spacecraft locations and ESW propagation characteristics for the six documented cases near the MPBL. This table provides, by column left to right, the following information: Date of cross spacecraft propagation detection and location; time of detection; two spacecraft on which the detection was made; lag time of detection of second spacecraft from the first as shown in previous column; pulse time duration of detected propagating ESWs; spacecraft separation parallel to the magnetic field; spacecraft separation perpendicular to the magnetic field; velocity of propagating ESWs; direction of propagation with respect to the earth; ESW size parallel to the magnetic field; and ESW size perpendicular to the magnetic field. Table 1 indicates that all of the ESWs except for the ones shown in Figure 1 are propagating toward the earth with velocities on the order of $1300-2400 \mathrm{~km} / \mathrm{s}$ and sizes parallel and perpendicular to the magnetic field of $0.5-1.0 \mathrm{~km}$ and $>$ than $40 \mathrm{~km}$, respectively. For comparison purposes for the event shown in Figure 1 (first event listed in Table 1), the electron thermal speed was $\sim 210 \mathrm{~km} / \mathrm{s}$ and the Debye length was on the order of $14 \mathrm{~m}$. Thus, the ESWs are propagating 
approximately 6.4 times faster than the thermal electrons and have a parallel size about 57 times larger than the Debye length.

\section{MODULATION OF ESWS}

On March 3, 2002, around 22:00 UT the Cluster spacecraft were moving inbound toward Earth and located in the magnetosheath, crossing the magnetopause into the cusp around 22:03 UT and on into the magnetosphere/polar cap at about 22:57 UT based on the CIS ion and PEACE electron data. The

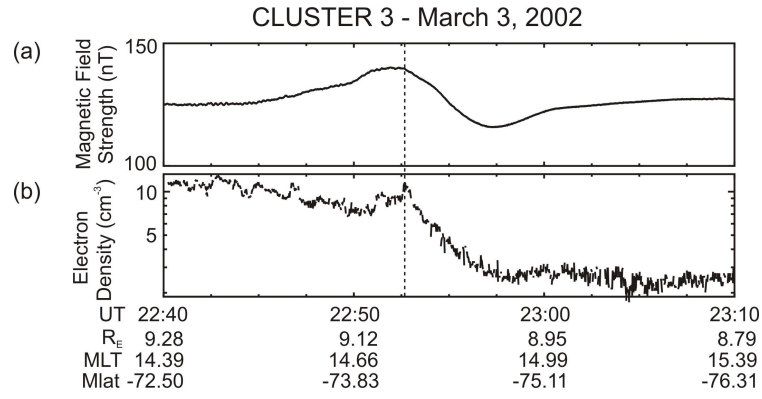

FIGURE 2. Magnetic field strength and density measurements on Cluster 3 while the spacecraft is in the magnetopause boundary layer on the earthward side. The dashed line indicates the time at which the data plotted in Figure 3 were obtained.

crossing of these two boundaries took place during the long recovery phase of a Co-rotating Interaction Region/High Speed Stream (CIR/HSS) event. Figure 2 shows the total magnetic field magnitude, in $\mathrm{nT}$, obtained from the FGM measurements (panel a) and the electron density obtained from Whisper measurements (panel b), both for Cluster spacecraft 3 (SC3) for the period 22:40 to 23:10 UT, thus encompassing the crossing into the polar cap from the magnetopause/cusp boundary at 22:57 UT. At the bottom of the figure is a listing of the spacecraft distance, $\mathrm{R}$ (in $\mathrm{R}_{\mathrm{E}}$ ), the magnetic local time, MLT (in hours), and the magnetic latitude, MLat (in degrees).

The focus of the current study is the time period 22:51 to 22:55 UT, during which time the modulated ESW bursts were detected almost continuously. In this period of time the electron density was $\sim 10 \mathrm{~cm}^{-3}$ and the magnetic field strength was $\sim 144 \mathrm{nT}$ as seen in Figure 2. Based on these values we obtain an electron plasma frequency $\mathrm{f}_{\mathrm{pe}} \sim 28.5 \mathrm{kHz}$, electron cyclotron frequency $\mathrm{f}_{\mathrm{ce}} \sim 4 \mathrm{kHz}$, proton cyclotron frequency $\mathrm{f}_{\mathrm{cH}^{+}} \sim 2.2 \mathrm{~Hz}, \mathrm{He}^{+}$cyclotron frequency, $\mathrm{f}_{\mathrm{cHe}^{+}} \sim 0.5 \mathrm{~Hz}$, and $\mathrm{O}^{+}$cyclotron frequency $\mathrm{f}_{\mathrm{cO}^{+}} \sim 0.14$ $\mathrm{Hz}$. At this time SC3 was located in the magnetopause/cusp boundary layer just prior to crossing into the polar cap and magnetosphere proper at $\sim 9 \mathrm{R}_{\mathrm{E}},-74$ degrees MLat and 14.7 hours MLT.

An example of the modulation of the ESWs observed during this period of time (dashed vertical line in Figure 2) is shown in Figure 3. Figure 3b shows wavelet analysis of the WBD waveform data using a Morlet mother wavelet, which strongly resembles the ESW pulses. The analyzed data cover an $\sim 2.4 \mathrm{~ms}$ time interval (horizontal axis) vs. frequency as inverse wavelet time scale (vertical scale) in the range $62 \mathrm{~Hz}$ to $10 \mathrm{kHz}$ with color indicating the power (intensity) of the waves in arbitrary units. We clearly see three bursts of electrostatic waves roughly in the band of $\sim 0.1$ to 4 $\mathrm{kHz}$. Note that the broad frequency, intense signatures (red color) observed just before 0.4 and $2.0 \mathrm{~s}$ and centered at $1.4 \mathrm{~s}$ are artifacts of gain change ringing in the WBD receiver and should be ignored. Figure $3 \mathrm{a}$ shows a representative $20 \mathrm{~ms}$ waveform taken from one of the bursts observed in Figure $3 b$. One of the ESW pulses, of which there are four that are well defined, in this example, has a pulse duration on the order of 300 microseconds and an amplitude of $\sim 0.64 \mathrm{mV} / \mathrm{m}$ peak-to-peak as indicated

(a)

(b)

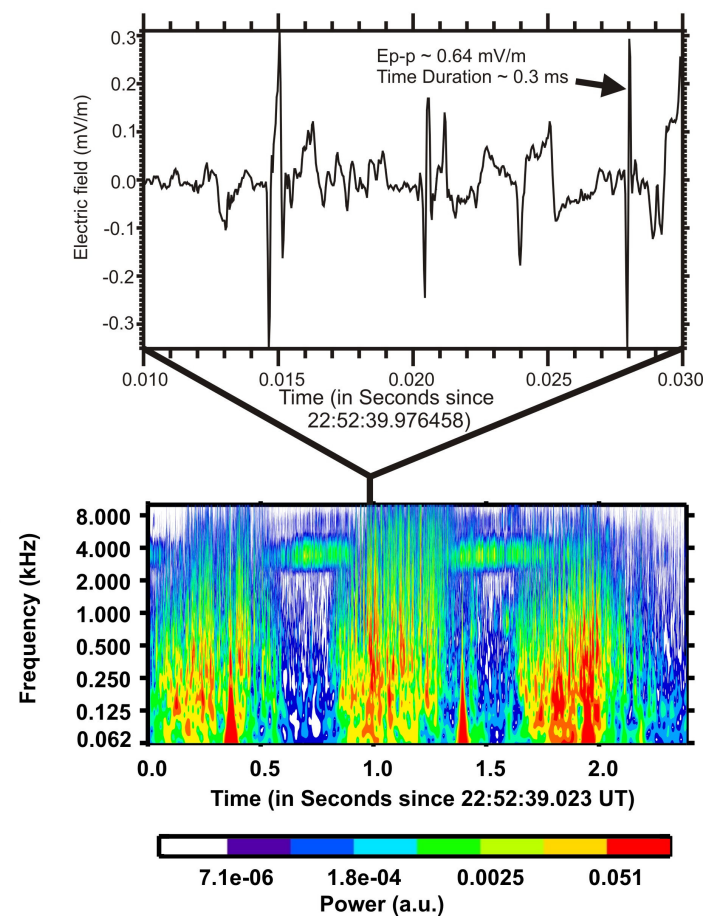

FIGURE 3. (a) Example of ESWs detected on Cluster 3. (b) Wavelet spectrogram showing bursts of electrostatic waves in the frequency range $0.1-4 \mathrm{kHz}$ and narrowbanded waves (probably whistler mode) around 3.5 - 4.0 $\mathrm{kHz}$. The electrostatic bursts are comprised of ESWs as shown by the location of the waveform sample in Figure 3(a). 
in Figure 3a. Pulses such as this make up the electrostatic bursts observed in Figure $3 b$ in the band of 0.2 to $4 \mathrm{kHz}$. These ESWs occur in bursts that are modulated at $\sim 1.3 \mathrm{~Hz}$ ( 3 bursts in 2.4 seconds) as opposed to being continuously or randomly observed throughout the $2.4 \mathrm{~s}$ time interval. We also note the presence of narrow-banded waves observed around 3.5 to $4.0 \mathrm{kHz}$ (just below $\mathrm{f}_{\mathrm{ce}}$ ). Based on the sinusoidal nature of these waves observed in the WBD waveforms and on STAFF-SA data analysis showing these waves to be right-hand polarized, they are most likely whistler mode waves given their frequency. They also appear to be modulated at the Pc1 wave frequency but out of phase with regard to the modulation of the ESW bursts.

Complementing these higher frequency wave observations are those of the STAFF-SC instrument onboard SC3 for the period from 22:45 to 23:00 UT in Figure 4. Panel a (top) shows the sum of the power spectral densities of the three components of the magnetic field. After filtering to retain the most energetic part of the spectrogram, we analyzed the wave properties with the singular value decomposition (SVD) method [17] as follows. Theta (in degrees), the angle between the wave vector $\mathrm{k}$ and the magnetic field $\mathbf{B}$, is shown in panel $b$ with the blue color code meaning $\mathbf{k}$ and $\mathbf{B}$ are parallel. The sense of polarization is plotted in panel $\mathrm{c}$ with the blue and red colors meaning left- and right-hand polarization, respectively (see equation (4) of Santolík et al. [18]). Ellipticity is shown in panel d with the blue and red colors meaning fully circular left- and right-handed waves, respectively. Panel e provides the coherency of the waves. A low coherency value [19] indicates that there are random phase shifts between the two components in the polarization plane. For each panel, frequency is plotted on the vertical scale, ranging from 0.5 to 3.5 $\mathrm{Hz}$, and the superimposed black line is the local proton cyclotron frequency.

The most striking feature observed in Figure 4, panel a, are the magnetic waves detected between 22:51 and 22:55 UT in the frequency range of about 1.2-1.7 Hz, which is greater than half of the local proton cyclotron frequency. Their intensity is larger than the background fluctuations by only about two orders of magnitude. Due to this relatively weak power and other electrostatic fluctuations, these waves are not well seen in the electric field data. Nevertheless, analysis of the Poynting flux using these data together with those shown in Figure 4 indicates that the energy of these waves is antiparallel to the magnetic field (not shown). Thus, these waves are propagating along the magnetic field from lower latitudes. The low theta values (the most frequent being $\sim 20-30$ degrees when the signal is

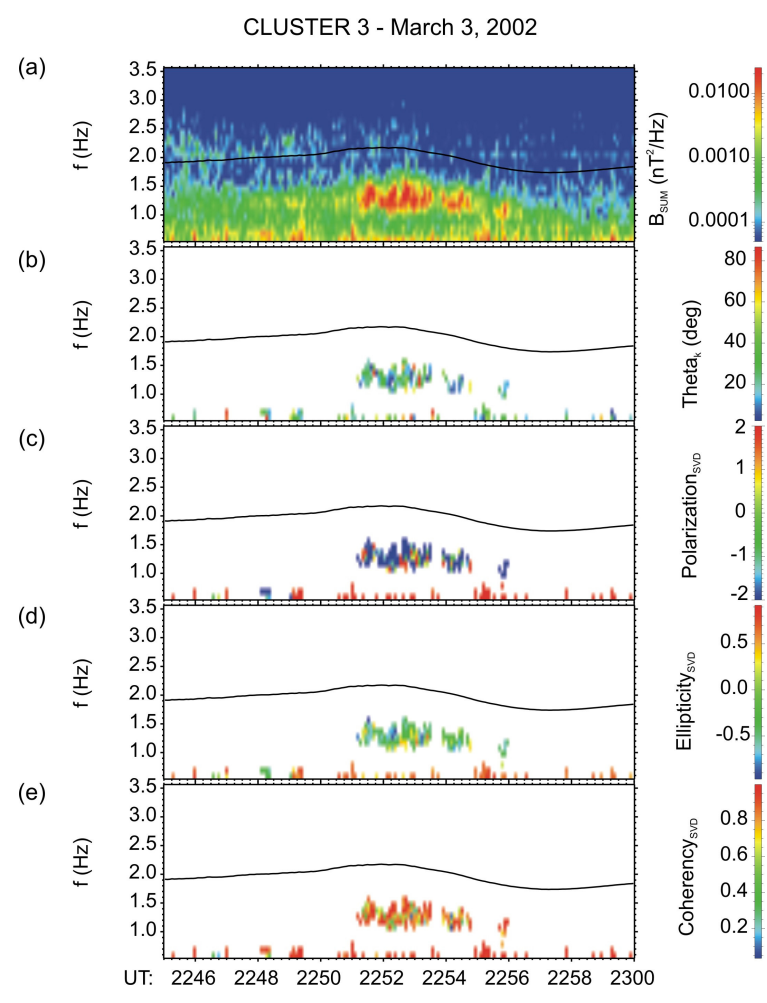

FIGURE 4: (a) Magnetic field spectral measurements in the frequency range 0.5 to $3.5 \mathrm{~Hz}$ showing the presence of Pc1 waves around $1.2-1.7 \mathrm{~Hz}$ from $22: 51$ to $22: 55 \mathrm{UT}$. (b) Theta angle of the $\mathrm{k}$ vector; (c) Polarization of the waves; (d) Ellipticity of the waves; and (e) coherency of the waves, using the SVD method as applied to the data plotted in panel (a)

strong) of these waves (panel b) indicate that their transverse component is larger than that of the compressive one. The sense of polarization is mainly left hand but with some right-hand excursions (panel c). This observation when considered with the low absolute value of the ellipticity (panel d) suggests the simultaneous presence of both left- and right-handed waves. Since the coherency level is quite high (panel e), the polarization analysis can be trusted.

Given the properties of these waves and their frequency range, they are most likely EMIC waves of the unstructured Pc1 type [20-22]. The Pc1 waves observed in Figure 4 are relatively weak compared to those from a similar ESW modulation event observed on March 30, 2002 near the plasmapause boundary layer (see [23]). Pc1 waves are expected to be present near the dayside magnetopause based on satellite and ground measurements [24-26]. Although there is still some uncertainty as to why these Pc1 waves are found there, there is no question as to their presence just inside the magnetopause on this date. Furthermore, although we have presented a 
single event of ESW modulation near the magnetopause, several other events exist within the Cluster data set but no attempt has yet been made to correlate these with the presence of Pc1 waves.

\section{DISCUSSION}

The ESW velocities as shown in Table 1 are consistent with propagation speeds of electron holes measured on single spacecraft in this region [6]. Their pancake or oblate shape (perpendicular scale with respect to the magnetic field much larger than the parallel scale) and characteristic size, which along the magnetic field is of the order of $1 \mathrm{~km}$ or less and across the field of tens of $\mathrm{km}$, are consistent with electron holes as well $[27,6]$. However, their characteristics are also consistent with electron acoustic solitons resulting from theoretical models for cusp and magnetosheath plasmas [5, 28]. Although we have been able to document propagation of ESWs across distances as great as tens of $\mathrm{km}$ near the magnetopause on the magnetosheath side by correlating a series of noncyclic ESW pulses across two different Cluster spacecraft, it was difficult and time-consuming to identify even the few cases shown in Table 1. Part of the difficulty is related to the fact that in most regions where ESWs are observed, i.e., boundary layers and turbulent regions, these regions are rich with other types of waves, some of which are of higher intensity, thus making it harder to isolate a series of ESWs for cross correlation analysis. Thus, the results of our cross spacecraft correlation study, shown in Table 1, are probably biased towards periods of time with lower wave intensity or activity and may not be applicable to more active magnetopause regions. Were we to cross correlate using only one isolated ESW pulse, we would have numerous correlations, almost all of which would be meaningless. This is because in any one region of space where ESW pulses are observed, they are usually numerous and of similar magnitudes and time duration as mentioned in Section 2. Obviously, the active physical processes which generate ESWs in any region of space at any given time will generate them with similar characteristics on an ongoing basis unless significant changes (currents, beams, density perturbations) in the plasma are introduced to either squelch those processes or change their characteristics due to those changes.

Our primary purpose in presenting results of propagation of ESWs was to demonstrate that nonlinear ESWs which are generated close to the magnetopause during periods of time in which wave activity is low to moderate can in fact be stable for as long as $22 \mathrm{~ms}$ ( 730 electron plasma periods), as opposed to the relatively short time of $\sim 0.1 \mathrm{~ms}$ observed on single spacecraft. This is a very long time considering the time scales of the ESWs themselves (few 100s of microseconds or $\sim 5-20$ electron plasma periods). Further, we have demonstrated that ESWs can propagate across great distances (tens of $\mathrm{km}$ ), in relation to their sizes along the propagation direction ( $1 \mathrm{~km}$ or less). This is perhaps an unexpected result and one which should be considered in the context of the results of Chen et al. [29] based on Polar data [4]. They found that the widths and amplitudes of electron holes are governed by an inequality relationship and are thus only loosely constrained in a system with a certain fluctuation level and different fluctuation lengths (i.e., they can exist within a large allowed region of width-amplitude space on one side of a bounding curve). Being loosely constrained allows them to be excited more easily since for a fixed electron hole amplitude, there is a wide range of allowed widths. This characteristic of loose constraint thus opens up the real possibility of spontaneous generation of long lasting phase-space holes in turbulent fluctuations. Hence, they are expected to play an important role in transport and electrical conductivity in collisionless plasma processes such as magnetic reconnection and boundary layer formation. It remains an open problem to prove that ESWs are playing a role in these processes, but having information on the propagation and stability of ESWs in these boundary regions will help shed light on that problem.

With regard to the modulation of ESWs, we have seen in Section 3 that co-located Pc1 waves at this modulation frequency are certainly essential in explaining the modulation phenomenon. Since the electron plasma period (few tens of microseconds) is usually of a time scale that is less than but consistent with the ESWs observed in the region of space near the magnetopause (few 100s of microseconds), we now look to the electron data for help in determining the generation of the ESWs that would also be consistent with their modulation. Figure 5 shows a cross section of the energy distribution function from the PEACE instrument which was recorded during the time of the ESW modulation bursts shown in Figure 3. The color scale shows the number of counts accumulated over the time interval shown with energy plotted on the vertical and horizontal axes. The magnetic field direction is up (or vertical) in this figure. Here we clearly see that electron beams of a few tens of $\mathrm{eV}$ are observed at parallel and anti-parallel to the magnetic field and at $\sim 45$ degrees from the anti-parallel direction. These beams are persistent throughout the time of the observations of the modulated ESWs and Pc1 waves 


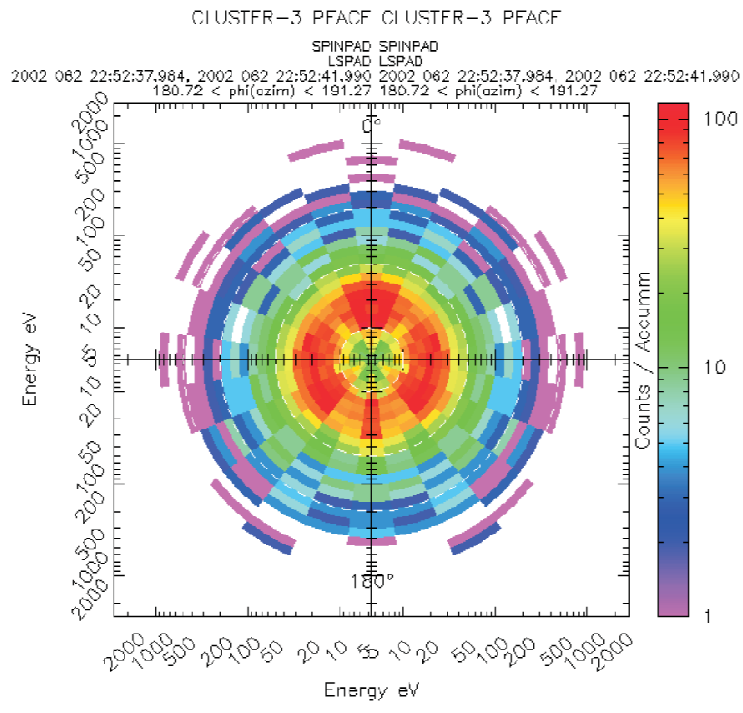

FIGURE 5. Cross section of the electron energy distribution function during the ESW measurements shown in Figure 3(a). Electron beams of a few tens of eV are present at parallel and antiparallel to the local magnetic field, as well as at 45 degrees to magnetic field direction.

( 2251 to $2255 \mathrm{UT}$ ), while outside this interval the electrons are more isotropic and/or at higher energies.

The ion data obtained from the CIS instrument are also remarkable in that in the period when the modulated ESW bursts are observed the direction of the ion velocity shows a direction reversal without undergoing a significant change in magnitude. The direction of flow of the $\sim 100 \mathrm{eV}$ protons undergoes a change primarily in the $\mathrm{V}_{\mathrm{x}}$ (GSE) direction from approximately $-30 \mathrm{~km} / \mathrm{s}$ to $+20 \mathrm{~km} / \mathrm{s}$ with the $\mathrm{V}_{\theta}$ angle changing from -160 to -45 degrees and the $V_{\varphi}$ from -55 to -85 degrees. It is possible that this change in direction is related to the presence of the Pc1 waves, just as the electron beams are present primarily during this same interval of time. It is also possible that the ion flow direction has changed in response to the d.c. magnetic field pulse seen in Figure 2 during this 22:51-:22:55 UT time period or that it is expected as the spacecraft approaches the polar cap boundary layer from the magnetopause/cusp region.

We are left with the question of how the Pc1 waves might be affecting the plasma environment close to the magnetopause such that ESWs are generated in bursts at the Pc1 wave frequency and what role the electrons and ions play in the generation of these ESWs. In order to answer this question, we first point out one very important aspect of the Pc1 waves. These waves will have an electric field component which lies along the magnetic field direction. This was previously demonstrated, for example, by Tsurutani et al. [30] using Polar data where an EMIC wave of similar frequency $(\sim 2.3 \mathrm{~Hz}$, slightly below the local $\mathrm{f}_{\mathrm{cp}}$ of $3.05 \mathrm{~Hz}$ ) was found to be propagating obliquely to the magnetic field in a polar cap boundary layer. This region is very similar to the one discussed here for Cluster. The EMIC wave in the Polar study was found to have a measurable $\mathrm{E}_{/ /}$component. This $\mathrm{E}_{/ /}$component can give rise to strong electric currents by accelerating electrons and ions in opposite directions. The accelerated electrons will appear as beams along the magnetic field. When the drift velocity of the electron beam exceeds the thermal velocity of the ions, the Buneman instability can be initiated [31]. If $\mathrm{T}_{\mathrm{i}} \gg \mathrm{T}_{\mathrm{e}}$, this instability will lead to formation of ESWs through the coalescence of large electrostatic potentials, while if $\mathrm{T}_{\mathrm{i}} \ll \mathrm{T}_{\mathrm{e}}$, ion acoustic waves will result. In the case under study, $T_{i}$ and $T_{e}$ are approximately of the same order, but in any case, not significantly different. In this case, the ESWs are still formed but the amount of trapped electrons will be less [31] and thus the process less efficient at generating ESWs. Particle simulations by Drake et al. [32] also showed that the Buneman instability developed in a current layer with subsequent formation of electron holes and particle energization during magnetic reconnection.

Lakhina et al. [33] suggested that for the Polar observations presented by Tsurutani et al. [30], the ESWs could have been generated through the bistream electron instability as discussed in Omura et al. [34]. The counter-streaming electron beams in this case arise through acceleration by the parallel electric field components of the obliquelypropagating ion cyclotron waves as suggested by Tsurutani et al. [30]. The analysis of Lakhina et al. [33] showed that such beam modes would saturate by trapping electrons, thus producing bipolar pulses and electron holes. We also look back to the GEOS observations of Cornilleau-Wehrlin [35] in which wide spectrum electrostatic waves which extended between the lower hybrid frequency and the ion plasma frequency were observed to be modulated at a ULF frequency around the helium gyrofrequency. The ULF-associated electrostatic waves were observed in the dayside region of the outer magnetosphere $(\mathrm{L}>6)$ at all geomagnetic latitudes sampled by the GEOS satellites (<30 degrees). Although the Cluster observations are at a much higher latitude, we believe that the phenomenon may be a similar one. The electrostatic waves observed by GEOS are clearly observed in a frequency range (hundreds of $\mathrm{Hz}$ ) much lower than Cluster (several $\mathrm{kHz}$ ). In addition, the waveforms were not available from which to determine whether ESW-type pulses 
were the primary constituent of those GEOS electrostatic waves. Nonetheless, Roux et al. [36] postulated that electrons having parallel velocities that are close to the Alfven velocity at the equator can be trapped in the potential troughs of the ULF waves due to the small but finite parallel electric field developed by the ULF waves in a multicomponent plasma. The trapped electrons are accelerated along the magnetic field as the parallel phase velocity of the ULF waves increase as they leave the magnetic equator. They suggest that the freshly detrapped electrons, which were found by Mauk and McPherron [37] to be modulated at the ULF frequency, are responsible for the ULFmodulated electrostatic waves. Thus, both the ULF waves and the ULF-modulated electrostatic waves can be observed simultaneously, through their connection with the accelerated electrons. It is beyond the scope of the present paper to carry out all of the necessary computations and develop models to determine the precise mechanism by which the Cluster ESWs are generated and modulated at the Pc1 wave frequency. However, as just discussed, the data support the suggestion that the $\mathrm{E}_{/ /}$component of the Pc1 waves initiates the process, setting up the conditions favorable for the Buneman instability to be excited. Furthermore, it is plausible to suggest that the trapped and accelerated electrons, which are due to the presence of the $\mathrm{E}_{/ /}$component of the Pc1 waves, could give rise to yet another type of instability in which modulated whistler mode waves are observed at a phase of the Pc1 wave other than that of the modulated ESW bursts.

We would like to end our discussion of modulated ESWs by comparing our results to laboratory and space-based observations of whistler mode wave packets. We begin with the ISEE-1 and ISEE-2 observation that whistler mode chorus emissions in the earth's outer magnetosphere on the dayside are often accompanied by high-frequency bursts of electrostatic waves whose frequencies were slightly below the electron plasma frequency. High time resolution measurements of these bursts reveal that at certain times these bursts are modulated at the chorus frequency. Reinleitner et al. [38] proposed a model in which the electrons are free to move along the ambient magnetic field line under the influence of the parallel electric field, $\mathrm{E}_{/ /}$, of the chorus waves. This electric field permits electrons to be trapped in effective potential wells of the chorus wave and to be carried along with them at the chorus phase velocity, thus exciting electrostatic waves via a two steam instability. A similar example on Polar in the cusp turbulent boundary of electrostatic bursts modulated at a whistler mode frequency was consistent with the proposed mechanism as that for the ISEE observations [39]. Both of these cases are consistent with the initial instability being that of the Buneman instability as discussed by Omura et al. [31] and with a high frequency instability discussed by Lakhina et al. [33] with regard to the generation of ESWs in the polar cap boundary layer from Polar observations and which involved the $\mathrm{E}_{/ /}$component of EMIC or Alfven waves. Recent laboratory experiments have been carried out in which a suprathermal electron beam was injected into a laboratory plasma resulting in the generation of ESWs and electrostatic whistler wave (EWW) packets [40]. The laboratory ESWs have time durations comparable to those derived from various observations of ESWs in the magnetosphere (e.g., [41]). Further, these EWW packets have similar characteristics to those of the electrostatic bursts discussed by Reinleitner et al. [38] and Pickett et al. [39], both of which are associated with whistler mode waves. Although the Cluster-modulated ESW bursts discussed here do not appear to be associated with certain phases of the whistler mode waves observed during the study event, but rather with a much lower frequency Pc1 wave similar to the Cornilleau-Wehrlin [35] and Roux et al. [36] events from GEOS, there is one common element tying all of these events together. All appear to depend on the $\mathrm{E}_{/ /}$component of an electromagnetic wave in the presence of the electrons, which allows the electrons to be accelerated and excite the Buneman instability.

\section{SUMMARY AND CONCLUSIONS}

We have shown that it is possible to detect ESW propagation across distances of a few tens of $\mathrm{km}$ using multi-spacecraft Cluster observations near the dayside magnetopause on the magnetosheath side. However, very few cases were found by using cross spacecraft waveform correlation techniques carried out on a computer with follow-up visual confirmation. The cases of high correlation are listed in Table 1 with their characteristic propagation speeds and sizes. These characteristics are similar to many electron holes and electron acoustic solitons previously reported in the literature, and help to confirm that our method for cross correlation is reliable. The primary conclusion we draw from this analysis is that ESWs can be stable over distances as great as tens of $\mathrm{km}$ and for time periods on the order of $22 \mathrm{~ms}$ and greater near the magnetopause. This is a very significant aspect of ESWs that needs to be considered when developing theories for how ESWs interact with particles and waves and modify the transport properties of the plasmas in which they are found. 
Our study of the modulation of the ESW bursts near the dayside magnetopause on the Earth side has found that these bursts occur at the same frequency as a concurrently observed Pc1 wave at $1.2-1.7 \mathrm{~Hz}$. This Pc1 wave will have a measurable electric field component parallel to the magnetic field, which over the time scale of the ESWs is nearly a d.c. electric field. This induced electric field allows for the acceleration of electrons and ions along the local magnetic field as the PC1 wave propagates, setting up the necessary conditions for the initiation of a Buneman type instability. This instability has been shown in previous theoretical and observational work to lead to the generation of ESWs, which would be modulated at the frequency of the wave that led to the initiation of the Buneman instability, i.e., the Pc1 waves for our event. Although we have presented data showing all of the necessary conditions are present to lead to these conclusions, it is an empirical result which needs to be investigated in more detail through theory and modeling. Furthermore, the possibility that most of the modulated ESW events reported in the literature in association with Pc1 waves observed in space are related to the modulated electrostatic wave bursts reported in the literature in association with whistler mode waves needs to be studied further since all cases seem to suggest that the $E_{/ /}$component for accelerating particles is common across all these cases. Finally, studying all of these events with those from the laboratory in which ESWs and EWWs are produced will help us in understanding the various generation mechanisms of ESWs and where these mechanisms are likely to be active in space.

\section{ACKNOWLEDGMENTS}

This work was supported at The University of Iowa by NASA GSFC under Grant NNX07AI24G and DOE grant DE-FG02-07ER54943, at the University of New Hampshire under DOE grant DEFG02-07ER54941, and at Augsburg College under NSF grant ATM-0827903. The Cluster Active Archive (CAA) is acknowledged for supplying the EFW low frequency electric field data referred to in this article. JSP thanks the organizing committee for inviting her to give a presentation of this work honoring the career of Dennis Papadopoulos at the conference "Modern Challenges in Nonlinear Plasma Physics". BG acknowledges Patrick Robert (LPP) for assistance in processing the STAFF-SC data. GSL thanks the Indian National Science Academy, New Delhi for support under the Senior Scientist Scheme. We acknowledge Elsevier for our partial reproduction of Figure 1.

\section{REFERENCES}

1. M. Temerin, K. Cerny, W. Lotko, and F. S. Mozer, Observations of double layers and solitary waves in the auroral plasma, Phys. Rev. Lett. 48, 1175-1179 (1982).

2. H. Matsumoto, H. Kojima, T. Miyatake, Y. Omura, M. Okada, et al., Electrostatic solitary waves (ESW) in the magnetotail: BEN wave forms observed by GEOTAIL, Geophys. Res. Lett., 21, 2915-2918 (1994).

3. J. S. Pickett, L.-J. Chen, S. W. Kahler, O. Santolík, D. A. Gurnett, D. A., et al., Isolated electrostatic structures observed throughout the Cluster orbit: Relationship to magnetic field strength, Annales Geophys., 22, 25152523 (2004).

4. J. R. Franz, P. M. Kintner, J. S. Pickett, L.-J. Chen, Properties of small amplitude electron phase-space holes observed by Polar, J. Geophys. Res., 110, A09212, doi:10.1029/2005JA011095 (2005)

5. S. S. Ghosh, J. S. Pickett, G. S. Lakhina, J. D. Winningham, B. Lavraud, B. and P. M. E. Décréau, Parametric analysis of positive amplitude electron acoustic solitary waves in a magnetized plasma and its application to boundary layers, J. Geophys. Res., 113, A06218, doi:10.1029/2007JA012768 (2008).

6. C. Cattell, J. Crumley, J. Dombeck, J. Wygant, and F. S. Mozer, Polar observations of solitary waves at the Earth's magnetopause, Geophys. Res. Lett., 29, 1065, 10.1029/2001GL014046 (2002).

7. H. Matsumoto, X. H. Deng, H. Kojima, and R. R. Anderson, Observation of electrostatic solitary waves associated with reconnection on the dayside magnetopause boundary, Geophys. Res. Lett., 30, 1326, doi:10.1029/2002GL016319 (2003).

8. D. A. Gurnett, R. L. Huff, and D. L. Kirchner, The Wide-Band Plasma Wave Investigation, Space Sci. Rev., 79, 195-208 (1997).

9. N. Cornilleau-Wehrlin, G. Chanteur, S. Perraut, L. Rezeau, P. Robert, et al., First results obtained by the Cluster STAFF experiment, Ann. Geophys., 21, 437456 (2003).

10. A. Balogh, M. W. Dunlop, S. W. Cowley, D. J. Southwood, J. Thomlinson, J., et al.: The Cluster Magnetic Field Experiment, Space Sci. Rev., 79, 65-91 (1997).

11. P. M. E. Décréau, P. Fergeau, V. Krannosels'kikh, M. Lévêque, Ph. Martin, et al., Whisper, A resonance sounder and wave analyzer: performances and perspectives for the Cluster mission, Space Sci. Rev., 79, 157-193 (1997).

12. A. D. Johnstone, C. Alsop, P. J. Carter, A. J. Coates, A. J. Coker, et al., PEACE: A plasma electron and current experiment, Space Sci. Rev., 79, 351-398 (1997).

13. H. Rème, C. Aoustin, J. M. Bosqued, I. Dandouras, B. Lavraud, et al., First multispacecraft ion measurements in and near the Earth's magnetosphere with the identical Cluster ion spectrometry (CIS) experiment, Ann. Geophys., 19, 1303-1354 (2001).

14. J. R. Franz, P. M. Kintner, and J. S. Pickett, POLAR observations of coherent electric field structures, Geophys. Res. Lett., 25, 1277-1280 (1998). 
15. J. S. Pickett, S. W. Kahler, L.-J. Chen, R. L. Huff, O. Santolík, et al., Solitary waves observed in the auroral zone: the Cluster multi-spacecraft perspective, Nonlin. Processes Geophys., 11, 183-196 (2004).

16. J. S. Pickett, L.-J. Chen, R. L. Mutel, I. W. Christopher, O. Santolík, O., et al., Furthering our understanding of electrostatic solitary waves through Cluster multispacecraft observations and theory, $A d v$. Space Res., 41, 1666-1676 (2008).

17. O. Santolik, M. Parrot, and F. Lefeuvre, Singular value decomposition methods for wave propagation analysis, Radio Sci., 38(1), 1010, doi:10.1029/2000RS002523 (2003).

18. O. Santolik, F. Lefeuvre, M. Parrot, and J. L. Rauch, Complete wave-vector directions of electromagnetic emissions: Application to INTERBALL-2 measurements in the nightside auroral zone, $J$. Geophys. Res., 106, 13,191-13,201 (2001)

19. O. Santolik and D. A. Gurnett, Propagation of auroral hiss at high altitudes, Geophys. Res. Lett., 29(10), 1481, doi:10.1029/2001GL013666 (2002).

20. J. A. Jacobs, Y. Kato, S. Matsushita, and V. A. Troitskaya, Classification of geomagnetic micropulsations, J. Geophys. Res., 69, 180 (1964).

21. K. Hayashi, T. Kokubun, T. Oguti, K. Tsuruda, S. Machida, T. Kitamura, O. Saka, and T. Watanabe, The extent of Pc1 source region in high latitudes, Can. J. of Phys., 69, 1097-1105 (1981).

22. B. J. Fraser, W. J. Kemp, and D. J. Webster, Pc1 pulsation source regions and their relationship to the plasmapause, Eur. Space Agency Spec. Publ., ESA SP217, 609-613 (1984).

23. J. S. Pickett, B. Grison, Y. Omura, M. J. Engebretson, I. Dandouras, A. Masson, M. L. Adrian, O. Santolik, P. M. E. Decreau, N. Cornilleau-Wehrlin, and D. Constantinescu, Cluster observations of EMIC triggered emissions in association with Pc1 waves near Earth's plasmapause, Geophys. Res. Lett., 37(9), doi:10.1029/2010GL042648 (2010).

24. B. J. Anderson, R. E. Erlandson, and L. J. Zanetti, A statistical study of Pc 1-2 magnetic pulsations in the equatorial magnetosphere 1. Equatorial occurrence distributions, J. Geophys. Res., 97, 3075-3088 (1992).

25. B. J. Anderson, E. R. Erlandson, M. J. Engebretson, J. Alford, and R. L. Arnoldy, Source region of 0.2 to 1.0 $\mathrm{Hz}$ geomagnetic pulsation bursts, Geophys. Res. Lett., 23, 769-772 (1996).

26. D. A. Neudegg, B. J. Fraser, F. W. Menk, G. B. Burns, R. J. Morris, and M. J. Underwood, Magnetospheric sources of Pc1-2 ULF waves observed in the polar ionospheric waveguide, Antarctic Science, 14(1), 93103 (2002).

27. J. R. Franz, P. M. Kintner, C. E. Seyler, J. S. Pickett, and J. D. Scudder, On the perpendicular scale of electron phase-space holes, Geophys. Res. Lett., 27, 169-172 (2000).

28. G. S. Lakhina, S. V. Singh, A. P. Kakad, M. L. Goldstein, A. F. Viñas, and J. S. Pickett, A mechanism for electrostatic solitary structures in the Earth's magnetosheath, J. Geophys. Res., 114, A09212, doi:10.1029/2009JA014306 (2009).
29. Li-Jen Chen, Jolene Pickett, Paul Kintner, Jason Franz, and Donald Gurnett, On the width-amplitude inequality of electron phase space holes, J. Geophys. Res., 110, A09211, doi:10.1029/2005JA011087 (2005).

30. B. T. Tsurutani, B. Dasgupta, J. K. Arballo, G. S. Lakhina, and J. S. Pickett, Magnetic field turbulence, electron heating, magnetic holes, proton cyclotron waves, and the onsets of bipolar pulse (electron hole) events: a possible unifying scenario, Nonlin. Processes Geophys., 21, 27-35 (2003).

31. Y. Omura, W. J. Heikkila, T. Umeda, K. Ninomiya, and H. Matsumoto, Particle simulation of plasma response to an applied electric field parallel to magnetic field lines, J. Geophys. Res., 108, 1197, doi:10.1029/2002JA009573 (2003).

32. J. F. Drake, M. Swisdak, C. Cattell, M. A. Shay, B. N. Rogers, and A. Zeiler, Formation of electron holes and particle energization during magnetic reconnection, Science, 299, 873-877 (2003).

33. G. S. Lakhina, B. T. Tsurutani, and J. S. Pickett, Association of Alfvén waves and proton cyclotron waves with electrostatic bipolar pulses: magnetic hole events observed by Polar, Nonlin. Proesses. Geophys., 11, 205-213 (2004).

34. Y. Omura, H. Matsumoto, T. Miyake, and H. Kojima, Electron beam instabilities as generation mechanisms of electrostatic solitary waves in the magnetotail, $J$. Geophys. Res., 101, 2685-2697 (1996).

35. N. Cornilleau-Wehrlin, A new ULF-modulated electrostatic wave detected in the extremely low frequency range onboard Geos, J. Geophys. Res., 86, 1365-1373 (1981).

36. A. Roux, N. Cornilleau-Wehrlin, and J. L. Rauch, Acceleration of thermal electrons by ICW's propagating in a multicomponent magnetospheric plasma, J. Geophys. Res., 89, 2267-2273 (1984).

37. B. H. Mauk and R. L. McPherron, An experimental test of the electromagnetic ion cyclotron instability with the earth's magnetosphere, Phys. Fluids, 23, 2111-2127 (1980).

38. Lee Reinleitner, Donald A. Gurnett, and Timothy E. Eastman, Electrostatic bursts generated by electrons in Laundau resonance with whistler mode chorus, $J$. Geophys. Res., 88, 3079-3093 (1983).

39. J. S. Pickett, J. R. Franz, J. D. Scudder, J. D. Menietti, D. A. Gurnett, G. B. Hospodarsky, R. M. Braunger, P. M. Kintner, and W. S. Kurth, Plasma waves observed in the cusp turbulent boundary layer: An analysis of high time resolution wave and particle measurements from the Polar spacecraft, J. Geophys. Res., 106, 19,081-19,099 (2001).

40. Bertrand Lefebvre, L.-J. Chen, W. Gekelman, P. Kintner, J. S. Pickett, P. Pribyl, S. Vincena, F. Chiang, and J. Judy, Laboratory measurements of electrostatic solitary structures generated by beam injection, Phys. Rev. Lett., submitted (2010).

41. J. S. Pickett, L.-J. Chen, O. Santolík, S. Grimald, B. Lavraud, et al., Electrostatic solitary waves in current layers: from Cluster observations during a supersubstorm to beam experiments at the LAPD, Nonlin. Processes Geophys., 16, 431-442 (2009). 